\title{
1:30 000 Cryogenic Current Comparator With Optimum SQUID Readout
}

\author{
Gert Rietveld, Elena Bartolomé, Javier Sesé, P. de la Court, Jaap Flokstra, Conrado Rillo, and Agustín Camón
}

\begin{abstract}
We have developed a 1:30000 winding ratio cryogenic current comparator (CCC) for measurement of small currents, e.g., generated by a single electron transport device. A dedicated low-noise SQUID is used for the readout, which allows reaching a sensitivity close to ideal, that is without loss of sensitivity in the flux transformer coupling the CCC and the SQUID. The CCC-SQUID equivalent current input noise using a 1:30 000 winding ratio is $2.1 \mathrm{fA} / \mathrm{Hz}^{1 / 2}$, measured down to $0.1 \mathrm{~Hz}$. Room temperature impedance measurements on CCCs appear to be a useful method for the determination of the screening by shields surrounding the $\mathrm{CCC}$ as well as for the detection of internal $\mathrm{CCC}$ resonances.
\end{abstract}

Index Terms-Cryogenic current comparator (CCC), current measurement, dc SQUID, inductance measurement, single electron transport (SET).

\section{INTRODUCTION}

$\mathbf{T}$ $\mathrm{HE}$ future establishment of a quantum standard for electrical current relies on the precise amplification of the very small quantized current ( 1 to $10 \mathrm{pA}$ ) provided by a single electron transport (SET) device, with the help of an ultra-sensitive, large-ratio cryogenic current comparator [1] with SQUID readout. The current quantum standard can then be combined with the existing resistance and voltage quantum standards to close the quantum metrological triangle of electrical units [2], [3]. The final current resolution of the cryogenic current comparator (CCC)-SQUID system (provided that all external sources of noise can be properly shielded) depends on the SQUID noise, and the efficiency of the coupling between the CCC and the SQUID. Usually, the CCC overlapped tube is coupled to the SQUID via a superconducting flux transformer. We have shown [4] that perfect coupling can be achieved connecting the tube directly to the SQUID input coil, in which case an optimal current noise is achieved

$$
\left\langle I_{P}^{2}\right\rangle^{1 / 2}=\frac{1}{N_{\mathrm{CCC}}} \sqrt{\frac{8 \varepsilon}{k_{\mathrm{sq}}^{2} L_{\mathrm{CCC}}}}
$$

Here, $\varepsilon$ is the intrinsic energy resolution of the SQUID, and $k_{\mathrm{sq}}$ is the coupling constant between the SQUID washer and input coil. To minimize $\left\langle I_{P}^{2}\right\rangle^{1 / 2}$, the CCC should have a large

Manuscript received June 17, 2002; revised October 31, 2002.

G. Rietveld and P. de la Court are with the NMi Van Swinden Laboratorium, Department of Electricity and Magnetism, Delft, The Netherlands (e-mail: GRietveld@NMi.nl).

E. Bartolomé, J. Sesé, and J. Flokstra are with the Low Temperature Division, Applied Physics Faculty, University of Twente, Enschede, The Netherlands.

C. Rillo and A. Camón are with the ICMA, CSIC-University of Zaragoza, 50009 Zaragoza, Spain.

Digital Object Identifier 10.1109/TIM.2003.809916

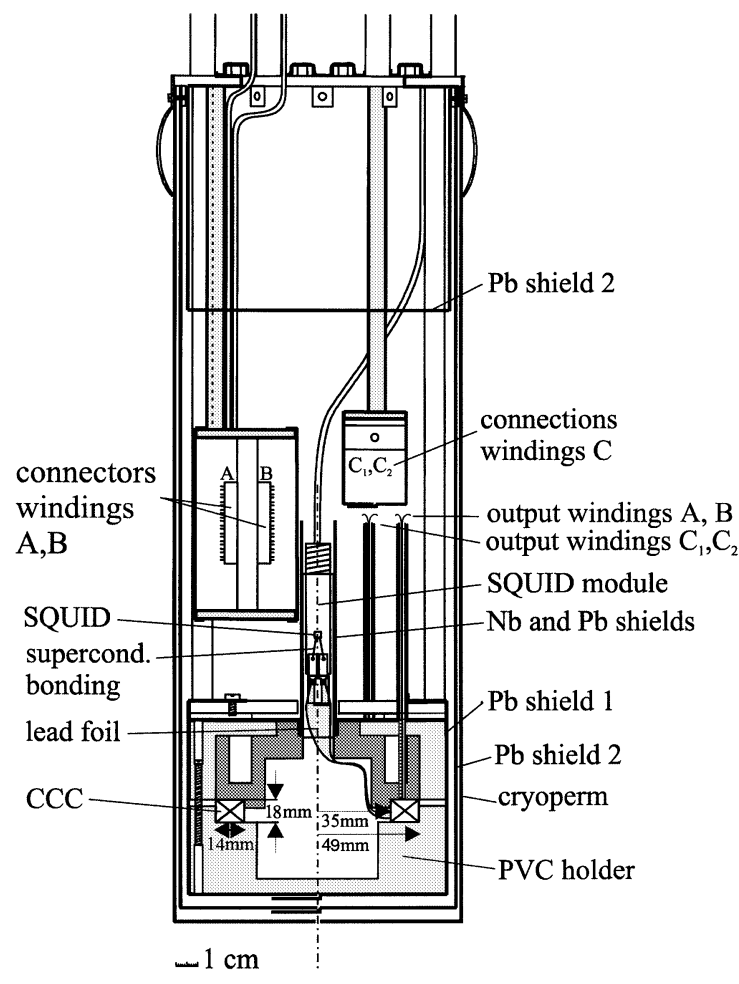

Fig. 1. Schematics of the lower part of the cryogenic insert containing the large ratio CCC with SQUID and the electrical and magnetic shields.

number of primary turns $N_{\mathrm{CCC}}$ and maximal self-inductance $L_{\mathrm{CCC}}$. Furthermore, the SQUID should have minimum energy resolution $\varepsilon$ and input coil inductance $L_{i}$ close to $L_{\mathrm{CCC}}$. In this paper, we present the integration and characterization of a complete 1:30000 CCC with optimal SQUID readout.

\section{EXPERIMENTAL SETUP}

A schematic view of the cryogenic part of the system is shown in Fig. 1 [5]. The CCC, with one turn overlap, contains the following sets of windings: $1,1,2,4,4,10,10,20,20,40,40,100$, $100,200,400,400,1000,1000,2000,4000,4000,10000$, and 10000 turns. Thus, the maximum amplification ratio available is 1:33 350. The windings are made of $70-\mu \mathrm{m}$-thick insulated $\mathrm{Cu}$ wire. The $\mathrm{CCC}$ has an inner diameter of $70 \mathrm{~mm}$ and a width and height of 14 and $18 \mathrm{~mm}$, respectively.

The CCC is completely surrounded by a Pb shield. Due to the image effect, the inductance $L_{\mathrm{CCC}}$ of the CCC becomes an effective inductance $L_{\mathrm{CCC} \text {,eff }}$ smaller than that of the unshielded CCC. The size of this effect is measured at room temperature with an LCR meter using a dummy $\mathrm{CCC}$ without windings and an overlapping tube of the same dimensions as that of 


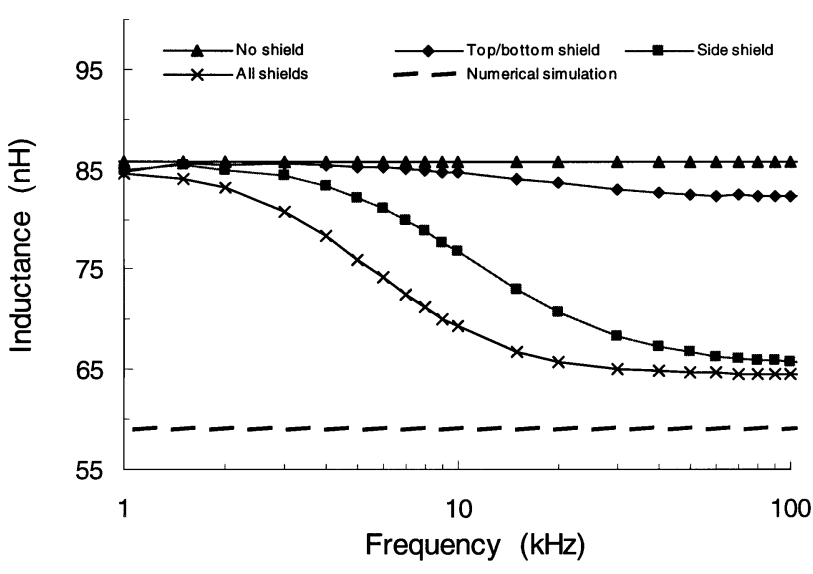

Fig. 2. Inductance of a dummy $\mathrm{CCC}$ without windings as a function of frequency for different configurations of the shields surrounding the CCC. The measurements were performed at room temperature using a commercial LCR meter.

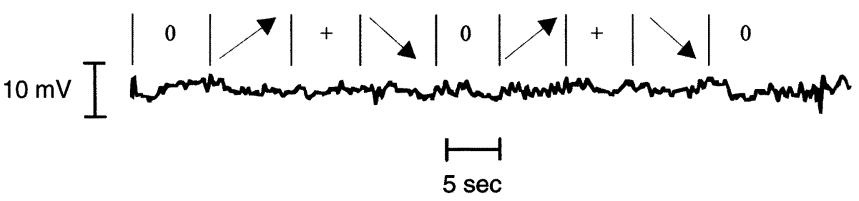

Fig. 3. SQUID output signal as a function of current applied to two 1000 turn windings placed in series with opposite polarity. The arrows indicate the up- and down-ramping of the $26-\mu \mathrm{A}$ current. No error signal is detected within the noise of about $10^{-4} \Phi_{o}$, which indicates a ratio error $F$ of less than $8.7 \times 10^{-9}$.

the 1:30000 CCC [6]. The results are given in Fig. 2, where the inductance values have been corrected for the zero of the LCR meter. The low frequency value of the inductance is that of the unshielded CCC whereas at frequencies above $10 \mathrm{kHz}, L_{\mathrm{CCC}}$ is affected by eddy currents in the surrounding shield. As expected, the effect of the side shield is larger than that of top and bottom shield. For the complete shield, the value measured by the LCR meter, $64 \mathrm{nH}$, compares very well with the numerically calculated value at $\mathrm{dc}$ for superconducting shields (all shields present) of $59 \mathrm{nH}$ [7].

A home-made $\mathrm{Nb} / \mathrm{Al}$ dc SQUID [5], [8] with input coil inductance $L_{i}=45 \mathrm{nH}$ close to $L_{\mathrm{CCC} \text {,eff }}$ is used. The noise of about $7 \mu \Phi_{o} / \mathrm{Hz}^{1 / 2}$ in the white noise region is dominated by that of the SQUID commercial readout electronics; at low frequencies, however, the noise is essentially that of the SQUID sensor and amounts only to approximately $11 \mu \Phi_{o} / \mathrm{Hz}^{1 / 2}$ at $1 \mathrm{~Hz}$. Annealed $\mathrm{Nb}$ wires, wedge bonded to the SQUID input coil pads, and spot welded to the SQUID module Nb blocks are used to connect SQUID input coil and mounting Nb blocks. A very low inductance $(<1 \mathrm{nH} / \mathrm{m})$ lead foil is used to connect the SQUID module to the CCC [6]. The SQUID module is shielded by two concentric $\mathrm{Nb}$ and $\mathrm{Pb}$ shields.

Only nonmagnetic materials are used in the construction of the CCC support system. The whole CCC-SQUID is fixed at the end of a rigid insert and surrounded by a second lead and cryoperm shield of an inner diameter of $135 \mathrm{~mm}$ and a height of $400 \mathrm{~mm}$ (Fig. 1). Finally, the complete cryostat is placed in a high-permeability iron can. The wires carrying small $\left(C_{1}, C_{2}\right)$ and high (A, B) currents are separated to avoid possible cross talk. Two $10-\mathrm{M} \Omega$ resistors are soldered in series with the $C_{2}$ winding to facilitate applying a small current. At room temperature, the $\mathrm{A}$ and $\mathrm{B}$ windings are available at two 20-pin Lemo connectors, while the $C_{1}, C_{2}$ windings are connected to triax connectors. A battery-powered current source is used to manually apply a variable current of either 0 to $26.4 \mu \mathrm{A}$ or 0 to $11.8 \mathrm{pA}$ to the windings.

\section{MeAsuREMENTS ON THE 1:30000 CCC}

First of all, the characteristics of the SQUID directly connected to the CCC has been measured. The period of the SQUID output voltage $V$ in the $V-\Phi$ curve corresponding to $1 \Phi_{o}$ of feedback flux is $5.5 \mathrm{~V}$, an intermediate situation between having the SQUID input coil leads open or shorted, giving 4.5 or $9 \mathrm{~V}$ per $\Phi_{o}$, respectively. In contrast to the normal direct $V-\Phi$ curve, the demodulated $V-\Phi$ curve is affected by superimposed large high-frequency signals. This behavior results from the appearance of LC-resonances in the multi-turn CCC, excited by the $500-\mathrm{kHz}$ modulation frequency signal of the SQUID readout electronics, that couples back to the flux transformer. The demodulated $V-\Phi$ curve and the noise spectrum thus contain all the excited resonant peaks corresponding to the different windings, with dominance of a peak at approximately $1.7 \mathrm{kHz}$ coming from the largest coil. The resonant phenomena make the system extra sensitive to vibrations and acoustic noise, but do not prevent the operation of the SQUID readout electronics in internal feedback mode.

The current sensitivity of the CCC is found to be $S_{\mathrm{CCC}}=$ $2.3 \mu \mathrm{A} \cdot \operatorname{turn} / \Phi_{o}$. This is close to the theoretical ideal value, being twice the current sensitivity of the SQUID of $1.1 \mu \mathrm{A} / \Phi_{o}$. The ratio error of the CCC has been measured by applying a current, reversed approximately every $5 \mathrm{~s}$ between 0 and $26.4 \mu \mathrm{A}$, to two 1000-turn windings connected in series opposition (see Fig. 3). No error signal can be detected within the SQUID noise of $10^{-4} \Phi_{o}$, which corresponds to a ratio error of less than $8.7 \cdot 10^{-9}[5]$.

The noise of the SQUID connected to the CCC as measured in flux locked loop (FLL) is given in Fig. 4 (upper curve). At high frequencies, the system noise spectrum shows the resonant behavior already commented, with the main peak at $1.7 \mathrm{kHz}$. The broad peak in the region of 10 to $100 \mathrm{~Hz}$ is due to vibrations. In this particular experiment, the noise in this area is extra large, due to the fact that several of the plastic screws clamping the CCC (Fig. 1) had been broken during cool down. The noise in this region is also large when several of the wires connected to the CCC windings are broken and thus are allowed to move relatively easily upon vibration excitation. The peak at $100 \mathrm{~Hz}$ is caused by the mains supply in the SQUID readout electronics and disappears when this electronics is run on battery supply. At frequencies lower than $10 \mathrm{~Hz}$, a flat noise level of about $27 \mu \Phi_{o} / \mathrm{Hz}^{1 / 2}$ is measured, corresponding to a noise current spectral density $\left\langle I_{P}^{2}\right\rangle^{1 / 2}$ (for a 1:30 000-turn winding ratio) of approximately $2.1 \mathrm{fA} / \mathrm{Hz}^{1 / 2}$. The $1 / \mathrm{f}$ noise of the SQUID (lower curve in Fig. 4) dominates below the corner frequency of about $0.1 \mathrm{~Hz}$, and it seems that connecting the CCC does not add significant $1 / f$ noise. To measure the noise at very low frequencies, the FLL output filtered at $1 \mathrm{~Hz}$ is recorded during $6 \mathrm{~min}$ and a current standard deviation of $\sigma_{I}=2.3 \mathrm{fA}$ was obtained. 


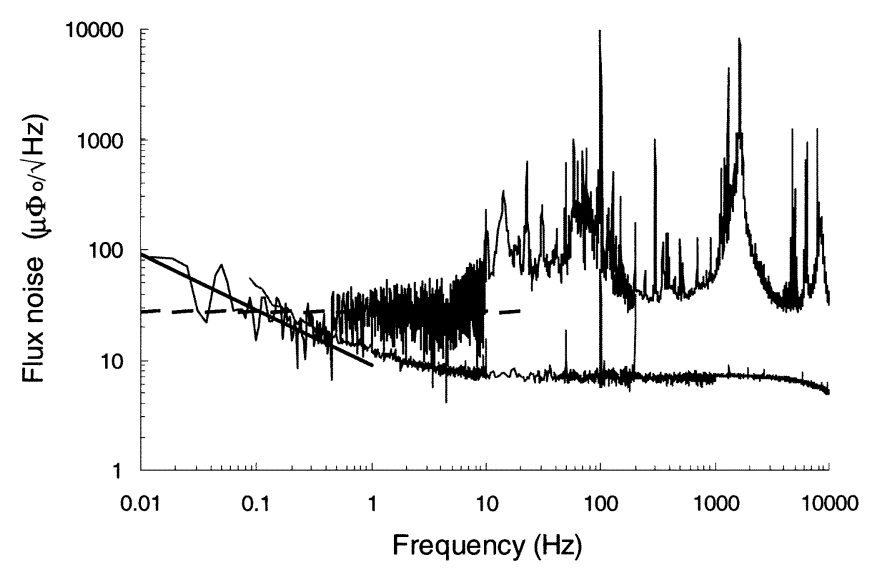

Fig. 4. Flux noise of the 1:30000 CCC (upper curve) and the bare SQUID (lower curve). The peak at $1750 \mathrm{~Hz}$ in the spectrum of the $\mathrm{CCC}$ is the internal resonance of the CCC; the broad peak in the region of 10 to $100 \mathrm{~Hz}$ is due to vibrations. The dotted line indicates the $27 \mu \Phi_{o} / \mathrm{Hz}^{1 / 2}$ noise of the CCC system in the 0.1 to $10 \mathrm{~Hz}$ range.

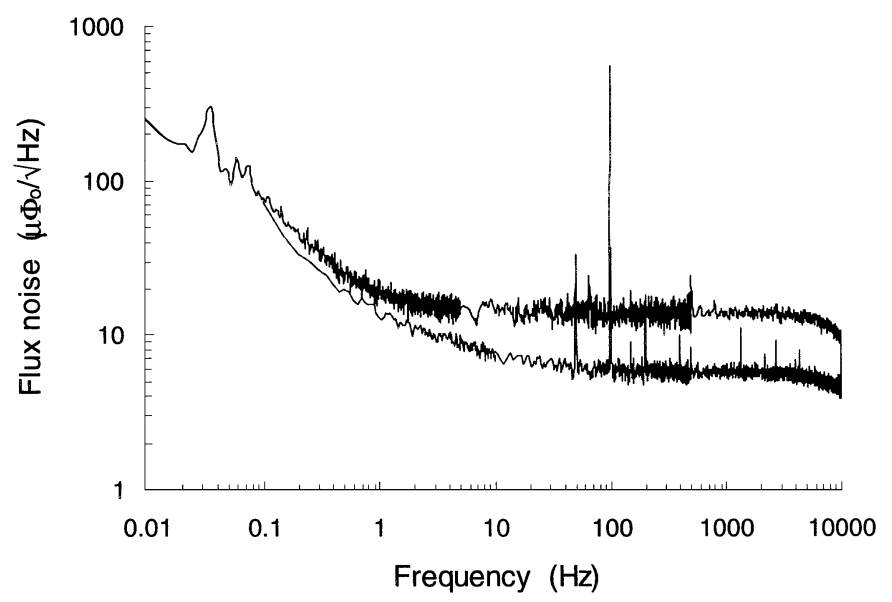

Fig. 5. Flux noise of the bare CCC (upper curve) compared with that of the bare SQUID (lower curve). The peaks at 50 and $100 \mathrm{~Hz}$ are caused by the SQUID readout electronics.

\section{MeAsurements on a Dummy CCC}

The accuracy of the final CCC setup is directly related to the noise as e.g., given in Fig. 4. Since the noise spectrum of the 1:30000 CCC shows the dominant presence of the internal resonance, we have performed a similar experiment with the dummy CCC used in the impedance measurements shown in Fig. 2. The result of the measurements is given in Fig. 5 and is compared to the spectrum of the bare SQUID. The spectrum is essentially smooth, with a peak at $100 \mathrm{~Hz}$ caused by the SQUID electronics and a small peak at $50 \mathrm{~Hz}$ from mains interference. The white noise is $15 \mu \Phi_{o} / \mathrm{Hz}^{1 / 2}$, which is only a factor two more than that of the bare SQUID.

The flux locked loop of the SQUID readout electronics is extremely stable during the measurements since the $\mathrm{CCC}$ has no windings inside. The low noise values and the clean noise spectrum indicate that our present electrical and magnetic shielding is sufficient. The extra noise seen in the noise spectrum might still be introduced by external sources or by the movement of frozen flux lines inside the CCC.

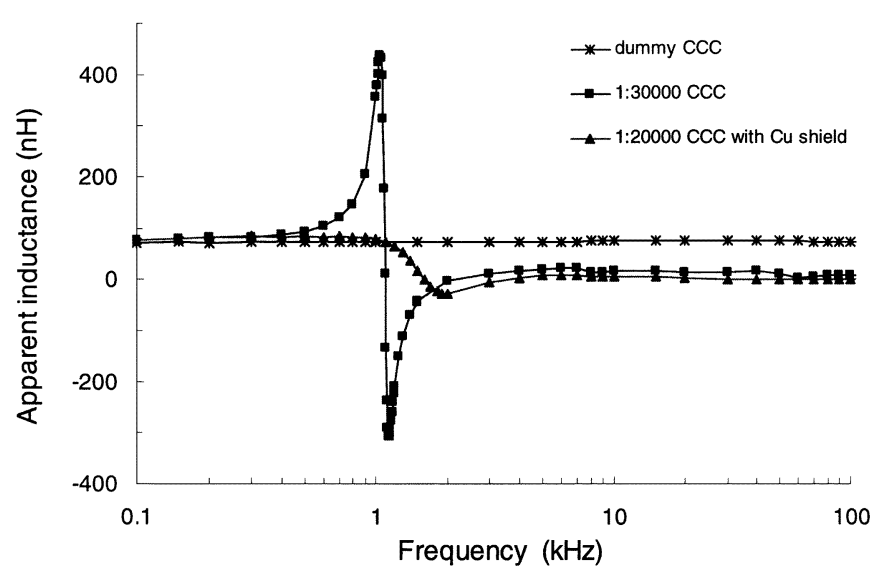

Fig. 6. Inductance of the three different CCCs examined in this study. The curve of the 1:30000 CCC shows a clear resonance peak at $1.1 \mathrm{kHz}$, whereas the $\mathrm{CCC}$ with internal $\mathrm{Cu}$ shields has a damped resonance around $1.4 \mathrm{kHz}$.

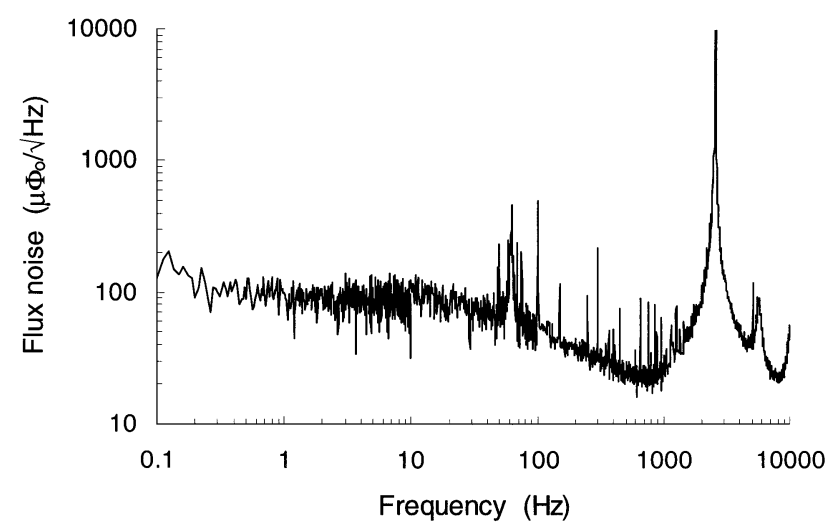

Fig. 7. Flux noise of the $\mathrm{CCC}$ with internal $\mathrm{Cu}$ shields. The internal resonance is still present at $2.5 \mathrm{kHz}$. The low frequency noise is relatively high and amounts to $90 \mu \Phi_{o} / \mathrm{Hz}^{1 / 2}$.

\section{Measurements on a CCC With InTERnal Cu SHIELDS}

The internal resonance of the 1:30000 CCC is a major source of extra noise and instability and therefore deserves further study. The resonance is caused by the large inductance of the 10000 windings, $L_{10000}=N^{2} \cdot L_{\mathrm{CCC}, \text { eff }} \approx 6 \mathrm{H}$, combined with its internal capacitance $C_{10000}$. Given the value of the resonance frequency and the value of $L_{10} 000$, we find a value of $C_{10000} \approx 1.4 \mathrm{nF}$. Similar values for internal capacitances were found by F. Gay [9]. In order to affect this internal resonance of the CCC, a 1:20000 winding ratio CCC was made where between every 2000 windings a single $\mathrm{Cu}$ layer was placed. Each $\mathrm{Cu}$ shield formed a 0.95 turn in order to not short the CCC transformer.

Fig. 6 shows the results of room temperature impedance measurements on all three CCCs, without shields. The dummy CCC gives an essentially constant value, as expected. The 1:30000 $\mathrm{CCC}$ has a clear resonance at $1.1 \mathrm{kHz}$, but the resonance of the 1:20000 CCC seems to be quite effectively damped by the internal $\mathrm{Cu}$ shields. Since the resonance of the 1:20000 CCC is around $1.4 \mathrm{kHz}$, the shields apparently do not significantly reduce $C_{\text {winding. }}$. This can be understood from the fact that the main capacitance contributing to $C_{\text {winding }}$ will be between successive layers of windings and there is only one $\mathrm{Cu}$ shield every 
8 layers of windings. All $\mathrm{Cu}$ shields were connected together, but isolated from the overlapping tube. The impedance spectrum did not change when the shields were grounded or not.

At $4.2 \mathrm{~K}$, using this CCC, the noise spectrum of Fig. 7 is obtained, from which it becomes clear that the resonance peak is still present at low temperatures. If the damping at room temperature is caused by eddy current dissipation in the $\mathrm{Cu}$ shields, this effect is smaller at low temperatures due to the lower resistance of the copper; it may be that other material, like, e.g., manganin, should have been used instead. Like for the impedance measurement, the noise spectrum does not depend on whether the $\mathrm{Cu}$ shields were grounded or not. The cause for the increase of noise below $700 \mathrm{~Hz}$ is still unclear. The noise levels there are much higher than what is found with the 1:30000 CCC. This will be the subject of future work.

\section{CONCLUSION}

We have developed a 1:30000 winding ratio CCC for the measurement of small (e.g., SET) currents. A dedicated lownoise SQUID is used for the readout, which allows reaching a sensitivity close to ideal, that is without loss of sensitivity in the flux transformer coupling the CCC and the SQUID. The CCC-SQUID equivalent current input noise using a 1:30000 winding ratio is $2.1 \mathrm{fA} / \mathrm{Hz}^{1 / 2}$, measured down to $0.1 \mathrm{~Hz}$. Measurements on a dummy CCC have revealed that the electrical and magnetic shielding of our cryogenic system is good, and that in the insert mechanical fixing of the wires connecting the CCC windings is very important. A test with a 1:20000 CCC with internal $\mathrm{Cu}$ shields did not result in lower current noise, even though at room temperature these shields damp effectively the internal resonances of the CCC.

Room temperature impedance measurements on CCCs appear to be a useful tool for the determination of the screening by shields surrounding the $\mathrm{CCC}$ as well as for the detection of internal $\mathrm{CCC}$ resonances.

We can estimate the current uncertainty that can be reached in the measurement of a SET current with our present 1:30000 $\mathrm{CCC}$, based on the measured noise current spectral $\left\langle I_{P}^{2}\right\rangle^{1 / 2} \approx$ $2.1 \mathrm{fA} / \mathrm{Hz}^{1 / 2}$. Assuming that the SET current can be reversed at a frequency $f_{S 1} \approx f_{c}=0.1 \mathrm{~Hz}$, assuming a measurement bandwidth of $0.1 \mathrm{~Hz}$ and an averaging over 720 measurement cycles ( $2 \mathrm{~h}$ measurement time), the standard deviation of the mean current would be $25 \mathrm{aA}$. In the measurement of a $1 \mathrm{pA}$ to $10 \mathrm{pA}$ SET current this would correspond to a relative uncertainty of approximately $3 \cdot 10^{-5}$ to $3 \cdot 10^{-6}$, respectively. If surface acoustic wave devices were available that can give accurate quantized currents of the order of $1 \mathrm{nA}$, a relative uncertainty less than $10^{-7}$ might be reached.

\section{REFERENCES}

[1] I. K. Harvey, "A precise low temperature dc ratio transformer," Rev. Sci. Instrum., vol. 43, pp. 1626-1629, 1972.

[2] F. Piquemal and G. Geneves, "An argument for the direct realization of the quantum metrological triangle," Metrologia, vol. 37, pp. 207-211, 2000 .
[3] E. Bartolomé et al., "SQUID based detectors in the electrical quantum metrology triangle realization," in Proc. 4th Workshop Low Temperature Electronics, Noordwijk, The Netherlands, 1999, pp. 177-183.

[4] J. Sesé et al., "Ultimate current resolution of a cryogenic current comparator," IEEE Trans. Instrum. Meas., vol. IM-48, pp. 1306-1313, Nov. 1972.

[5] E. Bartolomé, "Cryogenic Current Comparators With Optimum SQUID Readout for Electrical and Resistance Metrology," Ph.D., The Univ. Twente, The Netherlands, 2002.

[6] G. Rietveld et al., "Accurate measurement of small currents using a CCC with dc SQUID readout," Sens. Actuators A, vol. A85, pp. 54-59, 2000.

[7] J. Sesé et al., "Calculation of effective inductances of superconducting devices. Application to the cryogenic current comparator," IEEE Trans. Appl. Supercond., vol. 9, pp. 58-62, Jan. 1999.

[8] — "Low input coil inductance SQUID's for cryogenic current comparator applications," IEEE Trans. Appl. Supercond., vol. 9, pp. 3487-3490, June 1999.

[9] F. Gay, "Un Comparateur Cryogénique de Courants Pour la Réalization d'un Etalon Quantique Basé Sur L'effet Tunnel Monoélectron," Doctorat, Conservatoire National des Arts et Métiers, Paris, France, 2000.

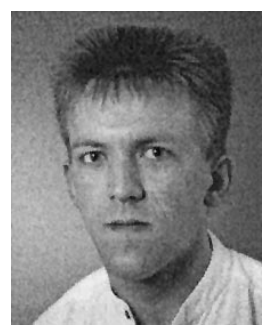

Gert Rietveld was born in The Netherlands in 1965. $\mathrm{He}$ received the M.Sc. and Ph.D. degrees in physics from the Delft University of Technology, Delft, The Netherlands, in 1988 and 1993, respectively.

In 1993, he joined the NMi Van Swinden Laboratorium, Delft, where he works in the DC/LF group of the Electrical Metrology Department. He is involved in the development of electrical quantum standards, especially the quantum Hall resistance standard and the current standard based on single electron tunnelling effects. He is also active in evaluation of "self-calibrating" instruments.

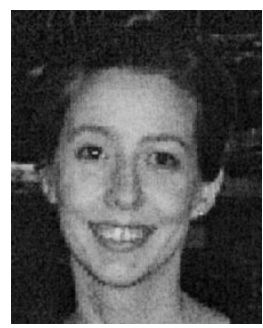

Elena Bartolomé was born in Zaragoza, Spain, on August 21, 1974. She received the M.Sc. degree in physics from the University of Zaragoza in 1997, and the $\mathrm{Ph} . \mathrm{D}$. degree in applied physics from the University of Twente, Enschede, The Netherlands, and the University of Zaragoza in 2002

She has worked on low Tc superconducting sensors (SQUIDs, cryogenic current comparator systems), with application in quantum metrology. At present, she has a post-doctorate position at the Institute of Material Sciences Barcelona (ICMAB), Barcelona, Spain, where she works on YBCO power applications and the development of chemically prepared coated conductors.

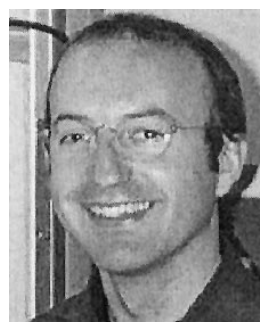

Javier Sesé is from Binéfar, Spain. He received the M.Sc. and Ph.D. degrees in physics from Zaragoza University, Zaragoza, Spain, in 1993 and 1999, respectively.

Since 1993, he has been with the Institute of Material Science of Aragon (ICMA), CSIC-University of Zaragoza. He has been working on electrical quantum metrology, applied superconductivity, and low-temperature instrumentation. Today, he is in a post-doctorate stay at the University of Twente, Enschede, The Netherlands, where he works on microelectromechanical systems for precision applications.

P. de la Court, photograph and biography not available at the time of publication. 


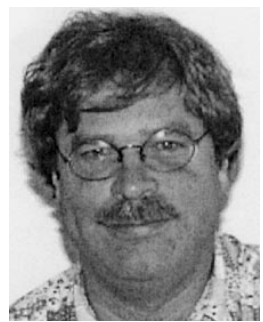

Jaap Flokstra received the M.Sc. degree in applied physics from the University of Delft, Delft, The Netherlands, and the Ph.D. degree in applied physics from the University of Twente, Enschede, The Netherlands, in 1971 and 1977, respectively.

He has been an Associate Professor in the Department of Applied Physics, University of Twente, since 1985. His current interest is in the development and application of low and high Tc SQUID-based sensors in areas as biomagnetism, particle detection, metrology, and very fast electronics.

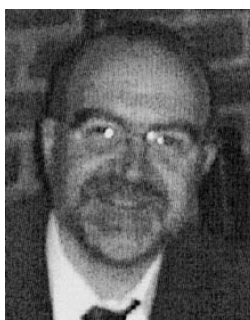

Conrado Rillo was born in Alcañiz, Spain, on May 8,1957 . He received the M.Sc. degree in physics and the Ph.D. degree from the University of Zaragoza, Zaragoza, Spain, in 1982 and 1986, respectively.

Since 1987, he has been with the research staff at the Institute of Material Science of Aragon (ICMA), CSIC-University of Zaragoza, where he has been working in the fields of materials research and advanced instrumentation. He is presently Research Professor and Head of the Low Temperature Physics Department at ICMA. His current research interests include magnetic and superconducting materials, quantum metrology and applied superconductivity. He is also responsible for the Scientific Instrumentation Service of the University of Zaragoza.

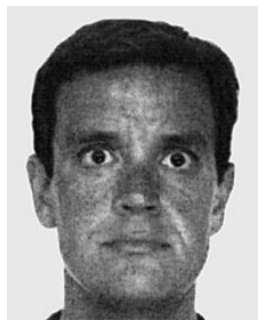

Agustín Camón was born in Peñaranda de Bracamonte, Spain, on March 17, 1962. He received the Ph.D. degree in physics from the University of Zaragoza, Spain, in 1996.

Since 1990, he has been with the Technical Staff at the Institute of Material Science of Aragon (ICMA), CSIC-University of Zaragoza, where he has been working on quantum metrology, applied superconductivity, and low temperature instrumentation. 